\title{
Fast and precise technique for magnet lattice correction via sine-wave excitation of fast correctors
}

\author{
X. Yang, V. Smaluk, ${ }^{*}$ L. H. Yu, Y. Tian, and K. Ha \\ National Synchrotron Light Source-II, BNL, Upton, New York 11973, USA
}

(Received 22 September 2016; published 2 May 2017)

\begin{abstract}
A novel technique has been developed to improve the precision and shorten the measurement time of the LOCO (linear optics from closed orbits) method. This technique, named AC LOCO, is based on sine-wave (ac) beam excitation via fast correctors. Such fast correctors are typically installed at synchrotron light sources for the fast orbit feedback. The beam oscillations are measured by beam position monitors. The narrow band used for the beam excitation and measurement not only allows us to suppress effectively the beam position noise but also opens the opportunity for simultaneously exciting multiple correctors at different frequencies (multifrequency mode). We demonstrated at NSLS-II that AC LOCO provides better lattice corrections and works much faster than the traditional LOCO method.
\end{abstract}

DOI: 10.1103/PhysRevAccelBeams.20.054001

\section{INTRODUCTION}

Linear optics from closed orbits (LOCO) [1] is a powerful beam-based diagnostics and optics control method for storage rings. LOCO is based on the measurement of the orbit response matrix (ORM). A small perturbation $\Delta x$ of the beam orbit is created by varying the strength of a corrector magnet located at the longitudinal position $s_{0}$ :

$$
\begin{aligned}
\Delta x(s)= & \Delta x^{\prime}\left(s_{0}\right) \sqrt{\beta_{x}\left(s_{0}\right) \beta_{x}(s)} \\
& \times \frac{\cos \left(\left|\psi_{x}(s)-\psi_{x}\left(s_{0}\right)\right|-\pi \nu_{x}\right)}{2 \sin \pi \nu_{x}},
\end{aligned}
$$

where $\Delta x^{\prime}$ is the transverse angle kick provided by the corrector, $\beta_{x}$ is the beta function, and $\psi_{x}$ and $\nu_{x}$ are the betatron phase and tune, respectively [2]. The orbit response vector $\Delta \mathbf{x}=\left[\Delta x_{1}, \Delta x_{2}, \ldots, \Delta x_{N}\right]$ to the corrector strength variation is measured by beam position monitors (BPMs), which are distributed around the ring. By repeating this process for every corrector in both horizontal and vertical directions, the ORM with $N \times M$ dimension is measured. Here $N$ is the number of BPMs, and $M$ is the number of correctors. Similarly, by varying the beam revolution frequency $f_{\text {rev }}$ with $\Delta f_{\text {rev }}$, the orbit deviation proportional to the dispersion function $\eta(s)$ at the BPM location can be also measured and included in the ORM as an $(M+1)$-st column:

$$
\Delta x_{\eta}(s)=-\frac{\eta(s)}{\alpha-\gamma^{-2}} \frac{\Delta f_{\text {rev }}}{f_{\text {rev }}} .
$$

\footnotetext{
Corresponding author. vsmalyuk@bnl.gov

Published by the American Physical Society under the terms of the Creative Commons Attribution 3.0 License. Further distribution of this work must maintain attribution to the author $(s)$ and the published article's title, journal citation, and DOI.
}

Here $\alpha$ is the momentum compaction factor [2], and $\gamma$ is the relativistic Lorentz factor. For NSLS-II, $f_{\text {rev }}=$ $378.546 \mathrm{kHz}, \frac{\Delta f_{\text {rev }}}{f_{\text {rev }}}=10^{-6}$, and the design momentum compaction is $\alpha=3.63 \times 10^{-4}$.

The measured ORM is then fitted to the model ORM by adjusting the model accelerator parameters, such as quadrupole and skew quadrupole strengths, and gains and rolls of the BPMs and correctors. Then the lattice model becomes a more accurate representation of the live machine; therefore, based on the fitting result, one can apply corrections to quadrupoles and skew quadrupoles to bring the machine closer to the design lattice.

The main disadvantage of the LOCO technique is that it takes a long time for the measurement and correction. The time varies from 10 up to 100 min depending on the size of the machine. As a result, LOCO suffers from systematic errors caused by slow drifts of machine parameters during the measurement, as well as by hysteresis effects of adiabatic (dc) variations of slow corrector magnets. Techniques based on turn-by-turn (TbT) BPM data processing [3] are much faster; however, they do not provide such high precision as LOCO, mainly due to the limited resolution of BPMs in the TbT mode.

In this article, we describe an AC LOCO technique based on a sine-wave beam excitation using fast orbit correctors; this approach was first reported in [4]. Using parallel computing, the authors of that paper have managed to reduce the time of diamond storage ring optics correction from $1 \mathrm{~h}$ to $5 \mathrm{~min}$. Introducing the multifrequency mode reduces the measurement time down to about a minute. The measurement time of dispersion function does not limit the achievable speed because it takes a small part of the total measurement time (about $1 / M$, where $M$ is the number of correctors). Here we present a detailed analysis of the noise suppression and accuracy limitations, and experimentally prove that $\mathrm{AC} \mathrm{LOCO}$ can provide a more 
precise ORM measurement and, therefore, better lattice correction, compared to the conventional LOCO. Finally, we compare the AC LOCO performance with the conventional LOCO as well as with TbT-based algorithms.

\section{MEASUREMENT TECHNIQUE}

At NSLS-II, 90 vertical and 90 horizontal fast orbit correctors have been installed for the fast orbit feedback [5]. For the ac ORM measurement, these correctors are used for a sine-wave beam excitation. Horizontal and vertical beam positions are measured simultaneously by 180 buttontype BPMs with a sampling frequency of $10 \mathrm{kHz}$ [6].

We are using a standard synchronous detection technique for the BPM data processing. The quadrature detection $(I / Q)$ technique is also applicable. In terms of accuracy, both techniques are equivalent but the synchronous detection is more convenient for digital data processing because it does not require a $90^{\circ}$ phase shift of the reference signal to produce $I$ and $Q$ components.

The ac driving signal (reference signal), which feeds a fast corrector, is described by

$$
U_{\text {ref }}(t)=a_{\text {ref }} \sin \left(\omega_{0} t+\varphi_{\text {ref }}\right),
$$

where $\omega_{0}$ is the excitation frequency, and $a_{\text {ref }}$ and $\varphi_{\text {ref }}$ are the excitation amplitude (kick angle) and phase of the reference signal, respectively. The beam response to the ac excitation of a fast corrector can be analytically described using a forced harmonic oscillator with damping. In our excitation frequency range, the damping effect results in a scaling factor of the corrector calibration, which is a LOCO fitting parameter; detailed analysis will be presented in the next section. Assuming the transfer function of the entire corrector-to-BPM circuit to be linear, the BPM signal can be written as

$$
U_{\mathrm{bpm}}(t)=a_{\mathrm{bpm}} \sin \left(\omega_{0} t+\varphi_{\mathrm{bpm}}\right)+\varepsilon(t) .
$$

Here, $a_{\mathrm{bpm}}$ and $\varphi_{\mathrm{bpm}}$ are the amplitude and phase of the BPM signal, and $\varepsilon(t)$ is the total noise of the system. The goal of the signal processing is to extract the amplitude $a_{\mathrm{bpm}}$ of the BPM signal, which is an element of the response vector $\Delta \mathbf{x}$. For this purpose, we consider the product $U_{\text {mix }}=U_{\text {ref }} U_{\mathrm{bpm}}$ of the reference (3) and BPM signal (4):

$$
\begin{aligned}
U_{\text {mix }}(t)= & \frac{1}{2} a_{\text {ref }} a_{\mathrm{bpm}} \cos \left(\varphi_{\mathrm{bpm}}-\varphi_{\mathrm{ref}}\right) \\
& -\frac{1}{2} a_{\mathrm{ref}} a_{\mathrm{bpm}} \cos \left(2 \omega_{0} t+\varphi_{\mathrm{bpm}}+\varphi_{\mathrm{ref}}\right) \\
& +\varepsilon(t) a_{\mathrm{ref}} \sin \left(\omega_{0} t+\varphi_{\mathrm{ref}}\right),
\end{aligned}
$$

where the first term is a dc component, and the second term is a double-frequency component. Averaging $U_{\text {mix }}(t)$ gives

$$
\begin{aligned}
\overline{U_{\text {mix }}} \equiv & \frac{1}{T} \int_{0}^{T} U_{\text {mix }}(t) d t \\
= & \frac{1}{2} a_{\text {ref }} a_{\mathrm{bpm}} \cos \left(\varphi_{\mathrm{bpm}}-\varphi_{\mathrm{ref}}\right) \\
& -\frac{a_{\mathrm{ref}} a_{\mathrm{bpm}}}{2 T} \int_{0}^{T} \cos \left(2 \omega_{0} t+\varphi_{\mathrm{bpm}}+\varphi_{\mathrm{ref}}\right) d t \\
& +\frac{a_{\mathrm{ref}}}{T} \int_{0}^{T} \varepsilon(t) \sin \left(\omega_{0} t+\varphi_{\mathrm{ref}}\right) d t .
\end{aligned}
$$

Here the third term is a spectral component of the noise at the oscillation frequency $\omega_{0}$. The second term in (6) is zero, if the measurement time $T$ is an integer number of oscillation periods. So the amplitude $a_{\mathrm{bpm}}$ can be easily found from the average $\overline{U_{\text {mix }}}$ :

$$
a_{\mathrm{bpm}}=\frac{2 \overline{U_{\mathrm{mix}}}}{a_{\mathrm{ref}} \cos \left(\varphi_{\mathrm{bpm}}-\varphi_{\mathrm{ref}}\right)}-\delta a,
$$

where $\delta a$ is the noise contribution coming from the third term of (6):

$$
\delta a=\frac{2 \int_{0}^{T} \varepsilon(t) \sin \left(\omega_{0} t+\varphi_{\mathrm{ref}}\right) d t}{T \cos \left(\varphi_{\mathrm{bpm}}-\varphi_{\mathrm{ref}}\right)} .
$$

Since the fast corrector provides a monochromatic sinewave excitation to the beam, only the noise in a very narrow band around $\omega_{0}$ contributes to the BPM signal and limits the BPM resolution. More detailed noise analysis will be discussed in the next section. The phase mismatch term $\cos \left(\varphi_{\mathrm{bpm}}-\varphi_{\text {ref }}\right)$ in the denominator, originating from the system delay, is just a constant factor and does not contribute to the measurement error, if it is time independent during the measurement.

\section{ACCURACY ANALYSIS}

The accuracy of the lattice measurement depends on several factors, such as the response functions of the fast correctors and their power supplies, the noise-limited resolution of the BPMs, systematic errors caused by slow drifts of the orbit and quadrupole power supply stability, and hysteresis of the corrector magnets. Since the fast correctors are driven by a sine wave, the magnets are automatically conditioned (hysteresis effect is removed). In addition, in the NSLS-II case the fast correctors are air-core and there is no hysteresis at all. Therefore, the advantage of the AC LOCO technique is that the effects of orbit drift and hysteresis on the measurement accuracy are negligible, and also the narrowband beam excitation allows us to efficiently suppress the beam position noise in the measurement.

To achieve better accuracy, it is important to choose the optimal frequency for the beam excitation, with the 


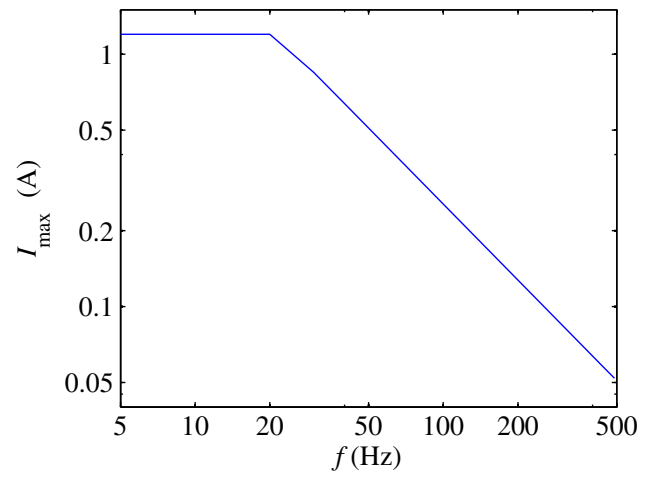

FIG. 1. The maximum power supply amplitude as a function of frequency.

maximum signal-to-noise ratio. The signal-to-noise ratio depends on the amplitude of the beam oscillation excited by a fast corrector magnet, the maximum field of which is determined by its power supply. For NSLS-II, the maximum ac amplitude of the power supply is shown in Fig. 1 as a function of frequency. Below $20 \mathrm{~Hz}$, it is determined by the current limit of $1.2 \mathrm{~A}$; above $20 \mathrm{~Hz}$, it is

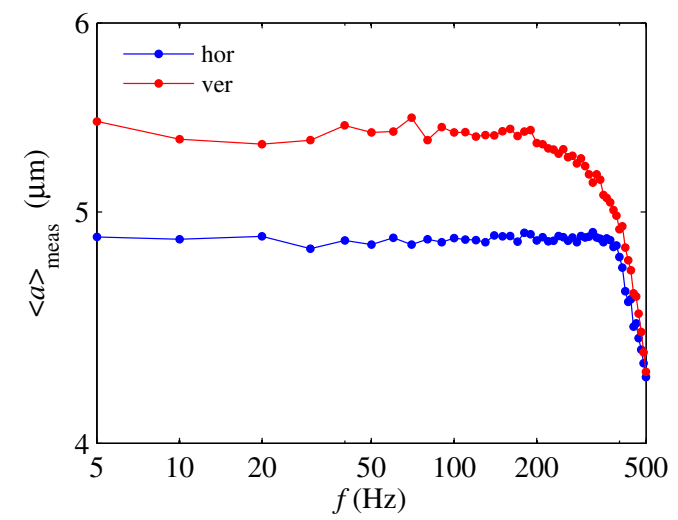

FIG. 2. Small-signal frequency response functions: horizontal (blue) and vertical (red). limited by the ramp rate of $160 \mathrm{~A} / \mathrm{s}$; therefore, we cannot effectively excite the beam at higher frequencies.

In order to characterize the entire corrector-to-BPM circuit including the power supply, the corrector magnet with vacuum chamber, the beam, and the BPM electronics, a small-signal frequency response function has been measured. The measured frequency responses are shown in Fig. 2. In the plot, $\langle a\rangle_{\text {meas }}$ is the BPM response (7) to a single-corrector ac excitation averaged over all BPMs. The excitation amplitude is $0.142 \mathrm{~A}$, which is about $12 \%$ of the maximum power supply current. The difference between horizontal and vertical response functions below $100 \mathrm{~Hz}$ is mainly determined by the lattice functions at the correctors and BPMs. So, the response to the ac excitation is linear up to $100 \mathrm{~Hz}$.

The noise-limited BPM resolutions have been estimated by analyzing power spectral density (PSD) of a series of 180 measurements without any beam excitation. The BPM data were measured during $T=5 \mathrm{sec}$; therefore the bandwidth is $\delta f=\frac{1}{T}=0.2 \mathrm{~Hz}$. One example of those 180 PSDs (square root) is shown in Fig. 3 (left); the gray area indicates the frequency range where PSD is averaged and the $\mathrm{BPM}$ noise is obtained via $\sqrt{\mathrm{PSD}\left(f_{0}\right) \cdot \delta f}$. According to Fig. 3, $\sqrt{\operatorname{PSD}(20 \mathrm{~Hz})} \approx 0.03 \mu \mathrm{m} / \sqrt{\mathrm{Hz}}$ and the BPM noise is about $0.013 \mu \mathrm{m}$. A separate measurement carried out by the NSLS-II beam diagnostic group gives very similar values of the BPM resolution [7]. The right plot in Fig. 3 shows an example of the beam oscillation spectrum measured by the same BPM, where the oscillation is excited by a single fast corrector at $20 \mathrm{~Hz}$. Using the data measured with and without beam excitation, we can estimate the signal-to-noise ratio.

Beam-based measurements of the frequency-dependent signal-to-noise ratio of the whole system have been carried out at the maximum available amplitude; the results are shown in Fig. 4 for horizontal (blue) and vertical (red) directions. According to the measurement results, the signal-to-noise ratio exceeds $50 \mathrm{~dB}$ in the whole range
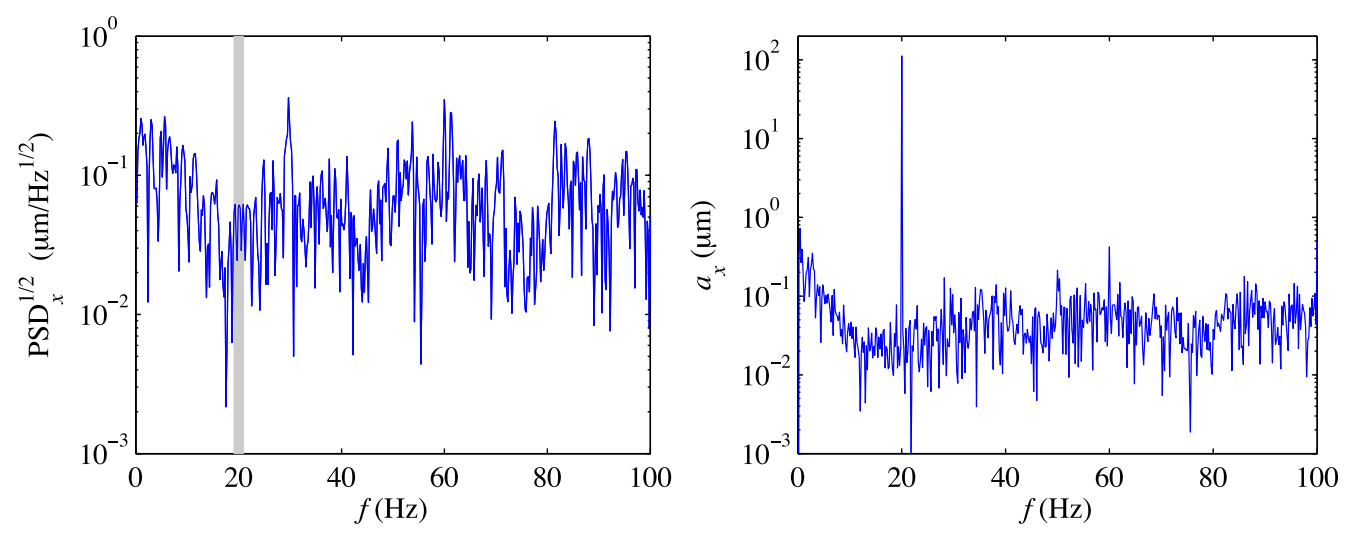

FIG. 3. Example of PSD spectrum of a horizontal BPM (left); example of beam oscillation spectrum (right). 


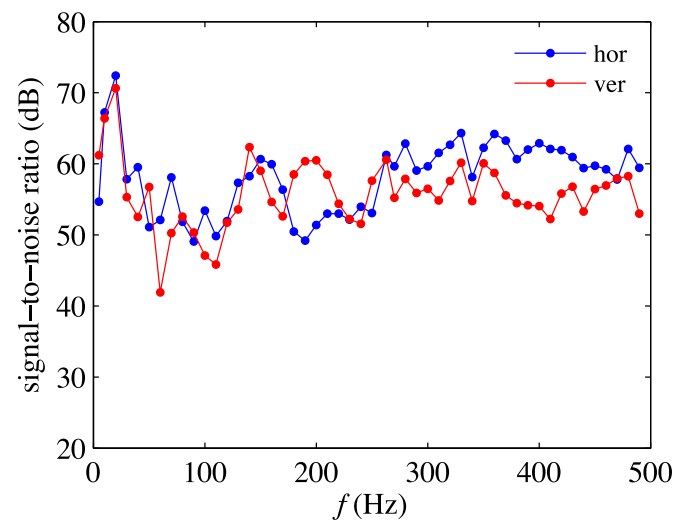

FIG. 4. Measured single-to-noise ratio vs frequency: horizontal (blue) and vertical (red).

except two areas around 60 and $100 \mathrm{~Hz}$. The optimal frequency for the best signal-to-noise ratio is around $20 \mathrm{~Hz}$.

Figure 5 shows the average noise-induced BPM errors within the measurement bandwidth of $0.2 \mathrm{~Hz}$ around $20 \mathrm{~Hz}$. Every point represents the average noise obtained from the power spectral density measured using 180 data

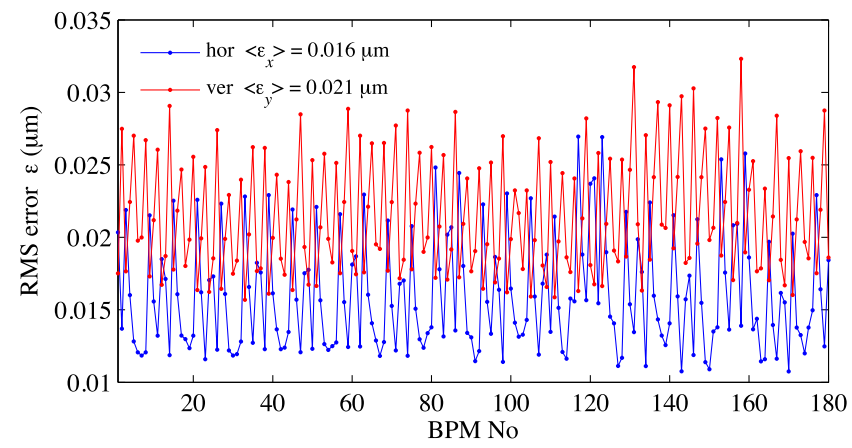

FIG. 5. Root mean square amplitude errors at $20 \mathrm{~Hz}$.

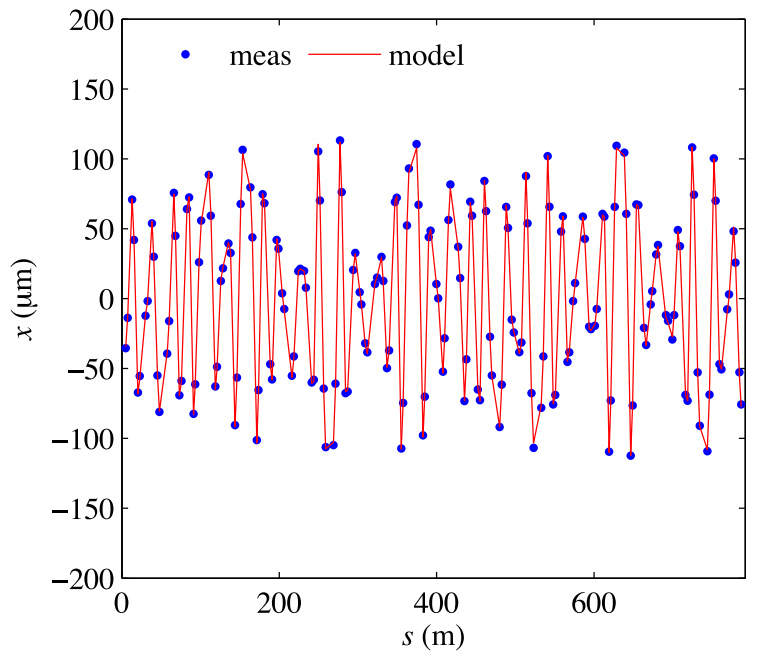

sets (see an example in Fig. 3). The horizontal and vertical BPM noise graphs show lattice-related patterns with the mean values $\left\langle\varepsilon_{x}\right\rangle=0.016 \mu \mathrm{m}$ and $\left\langle\varepsilon_{x}\right\rangle=0.021 \mu \mathrm{m}$. The total noise is dominated by the beam motion; the actual noise of BPM electronics is much smaller.

The amplitude of beam position oscillation measured by a BPM depends on the values of beta functions at the locations of the BPM and the corrector and on the betatron phase advance between them, according to (1). The BPM response to the $\mathrm{AC}$ excitation of a fast corrector is described by an equation of forced harmonic oscillator with a damping term. The general solution is a sum of the homogeneous part (decaying term) and the nonhomogeneous part (steady-state term). In our case, the decaying term vanishes because we delay taking any BPM data by a time much longer than the radiation damping time $(50 \mathrm{~ms}$ for NSLS-II) after starting the AC excitation. In the steadystate term, the damping influences the amplitude and phase of the response function as a scaling factor, which is absorbed by the LOCO fitting parameters (corrector calibrations). Examples of the horizontal (left) and vertical (right) oscillation amplitudes measured by all BPMs (blue), which corresponds to one column of the ORM (a single corrector), are presented in Fig. 6, together with the model data (red).

We numerically investigate how the BPM resolution influences the LOCO fitting results. We found that, if the $\mathrm{BPM}$ resolution is below $10 \mathrm{~nm}$, the precision of the linear lattice is mainly determined by the crosstalks between different fitting parameters in the LOCO fitting process. Since the measured noise-limited BPM resolution is close to this number, we can state that the accuracy of the linear lattice correction by LOCO has been improved to an extent that it is now mainly limited by the systematic errors, such as the orbit drift and quadrupole power supply fluctuation during the measurement, etc., not by the BPM resolution.

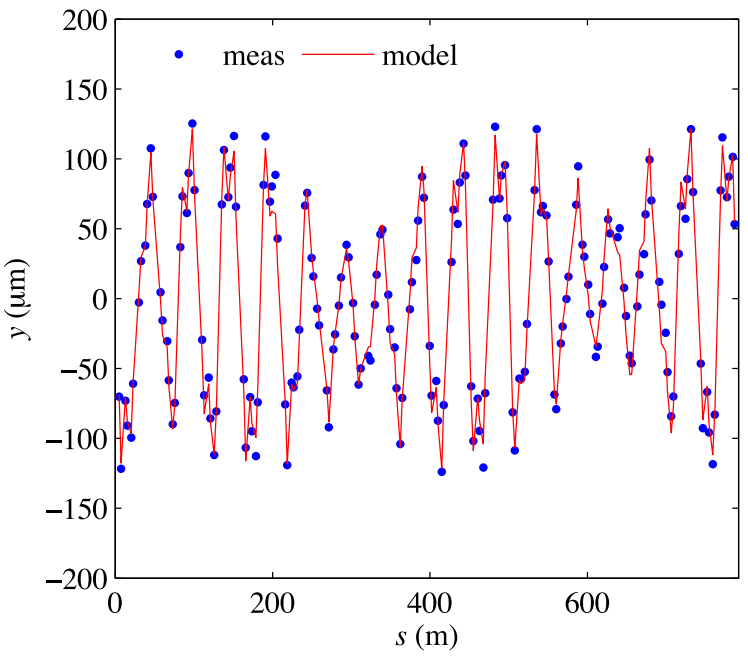

FIG. 6. Amplitudes measured by all BPMs at $20 \mathrm{~Hz}$ : horizontal (left) and vertical (right). 


\section{MULTIFREQUENCY EXCITATION}

Since the signal bandwidth is quite small $(0.2 \mathrm{~Hz}$ for a $5 \mathrm{sec}$ measurement), it provides a unique opportunity to simultaneously excite the beam oscillation via multiple correctors with different frequencies separated by an interval of $\Delta f$. This technique can potentially reduce the measurement time down to a minute, which is comparable to the TbT-based methods, also with at least an order of magnitude improved measurement precision.

How we choose the frequency range for the multiple excitations is mainly based on the measured frequencydependent signal-to-noise ratio of the system (Fig. 4). We can set the excitation frequency up to $100 \mathrm{~Hz}$ still having the beam oscillation amplitude acceptable for the linear lattice correction. Due to the hardware limitation at NSLS-II, only 30 frequency-separated signals are available at the same time for driving the fast correctors. Therefore, the frequency separation $\Delta f$ is decided to be less than $3 \mathrm{~Hz}$. However, $\Delta f$ has to be large enough to keep the crosstalk from adjacent excitations below the noise level.

The beam oscillation measured by BPMs is a finite-time sine wave, Fourier transform of which is proportional to the sinc function $\operatorname{sinc}(\pi T \Delta f) \equiv \frac{\sin \pi T \Delta f}{\pi T \Delta f}$, where $\Delta f=f-f_{0}$, $f_{0}$ is the excitation frequency. This function has zero values at $\Delta f=k / T$, where $k$ is an integer, so we can choose any of these frequencies for the multifrequency excitation. In our experiment, $T=5 \mathrm{~s}$ and $\Delta f=k \Delta 0.2 \mathrm{~Hz}$, we choose $\Delta f=2 \mathrm{~Hz}$. Figure 7 shows an example of the spectra of fast corrector current with excitation frequencies of 20 and $22 \mathrm{~Hz}$. As one can see, the interference is minimal, if we choose $\Delta f$ multiple of $0.2 \mathrm{~Hz}$.

We have done a proof-of-principle experiment with 23 ac driving signals available at the time being. The excitation frequencies are $10 \mathrm{~Hz}, 12 \mathrm{~Hz}, \ldots, 54 \mathrm{~Hz}$; see the spectra of the horizontal and vertical BPM signals (Fig. 8). We repeated the same measurement 10 times in both horizontal and vertical directions to estimate the statistical errors. For each set of the data $(j=1 \ldots 10)$, averaging all BPMs' response to every excitation

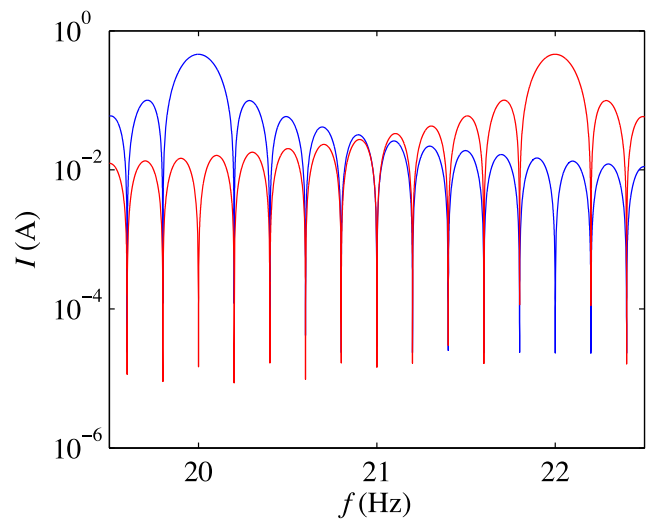

FIG. 7. Spectra of fast corrector excitation signals.

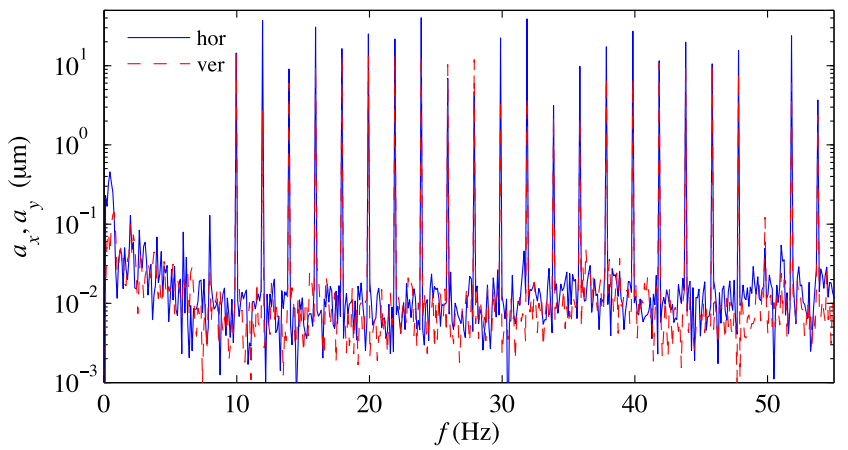

FIG. 8. Amplitude spectra of horizontal (blue) and vertical (red) BPM signals.

frequency $f_{i}(i=1 \ldots 23)$, we obtain $\left\langle a\left(f_{i}\right)\right\rangle_{j}$. The rms value over those 10 data sets at each excitation frequency $f_{i}$ gives the BPM resolution at $f_{i}$. In the measurement, we keep the slow orbit feedback on to minimize the slow beam motion. In the excitation frequency range from 10 to $54 \mathrm{~Hz}$, the measured errors are less than $20 \mathrm{~nm}$. We experimentally achieve similar BPM resolutions in the multifrequency mode compared to the single-frequency mode. The difference of the measured ORMs (multifrequency and single frequency) is also within the statistical errors. Therefore, no matter which mode AC LOCO is conducted, we should have similar performances of linear lattice corrections. We experimentally proved the feasibility of simultaneously exciting many horizontal (or vertical) fast correctors at different frequencies. At NSLS-II, we are limited by the hardware but not by the method itself. Based on this, we estimate that it would take less than $1 \mathrm{~min}$ for the ORM measurement.

\section{EXPERIMENTAL RESULTS}

The achieved resolution is sufficient to improve the precision of the lattice correction in comparison with the conventional LOCO and other algorithms based on TbT BPM data. To test the performance and limitation of the AC LOCO correction, we have applied the new technique to correct a lattice with large initial beta beat and coupling made by adding random errors to quadrupoles and skew quadrupoles. We have compared the performance of AC LOCO with the conventional LOCO as well as with four algorithms based on TbT BPM measurements. A crosscheck of the TbT-based algorithms (weighted correction of betatron phase and amplitude [8], independent component analysis [9], model-independent analysis [10], and driving-terms-based linear optics characterization [11]) has been done in the previous experiment at NSLS-II [3].

Figure 9 illustrates the convergence of the horizontal (left) and vertical (right) beta functions corrected iteratively using all these techniques. These are the residual rms errors 

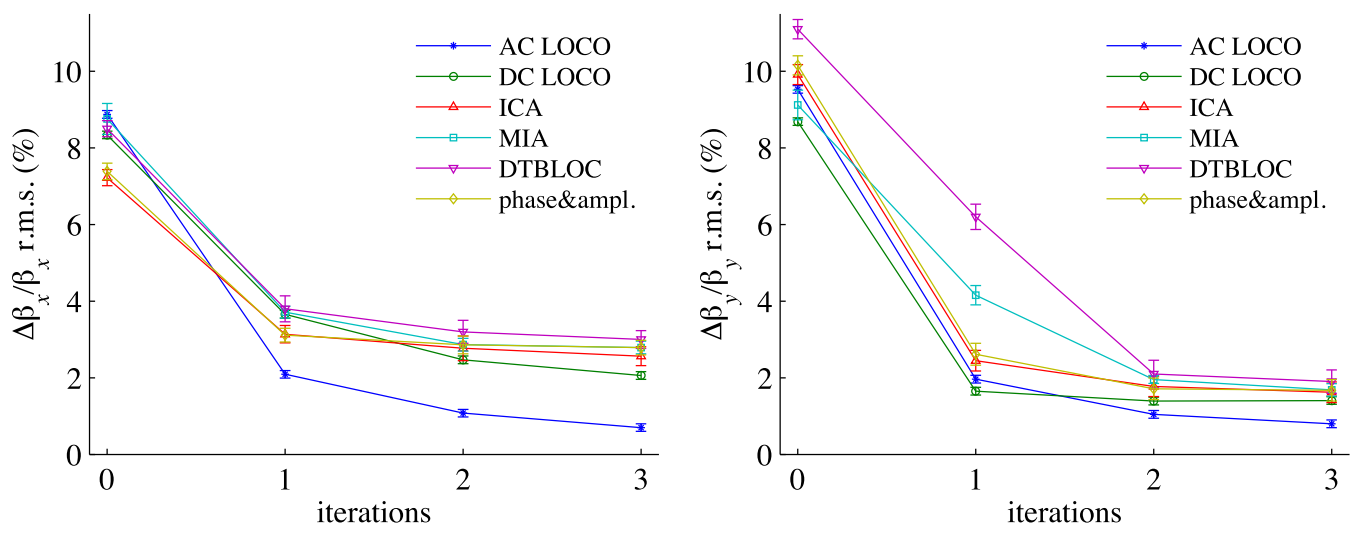

FIG. 9. Convergence of beta function: horizontal (left) and vertical (right).
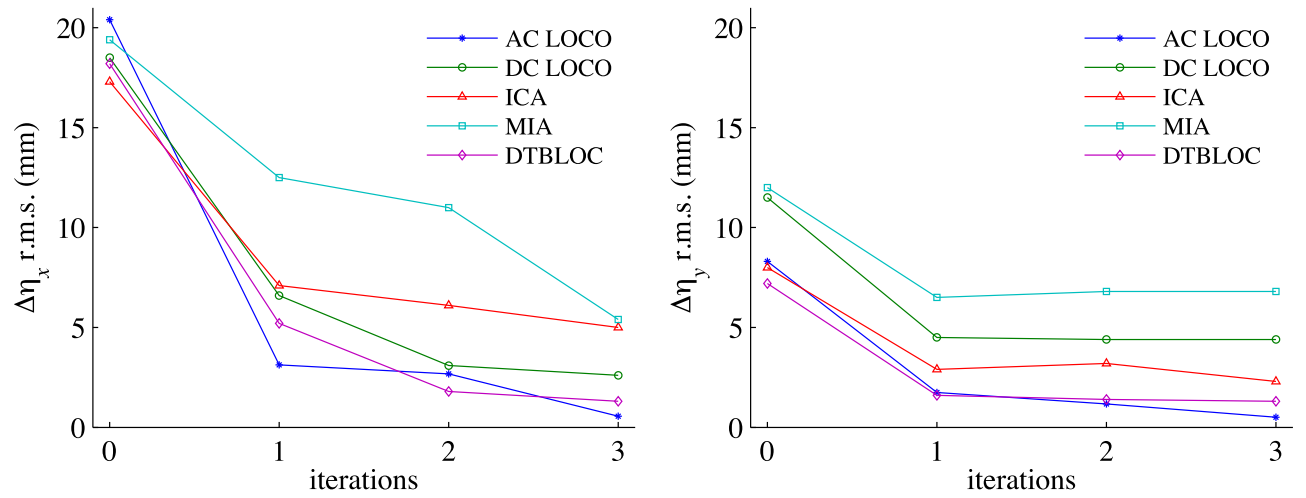

FIG. 10. Convergence of dispersion: horizontal (left) and vertical (right).

of beta functions plotted as functions of the number of iterations applied. The beta error is defined as $\frac{\beta_{\text {meas }}-\beta_{\text {model }}}{\beta_{\text {model }}}$, where $\beta_{\text {model }}$ is the beta function of the ideal lattice model, and $\beta_{\text {meas }}$ is the beta function obtained from the fitted model in both DC and AC LOCO cases. In the AC LOCO case, the fitting algorithm is exactly the same as the standard LOCO technique [1], except the ORM is measured via the $\mathrm{BPM}$ response to ac excitation of fast correctors. In TbTbased cases, $\beta_{\text {meas }}$ is obtained by spectral analysis of TbT BPM data [3]. As one can see, AC LOCO gives the best results; three iterations of the $\mathrm{AC} \mathrm{LOCO}$ correction reduce the beta-beating errors from $10 \%$ down to $0.7 \%$. This is at least a factor of two reduction compared with the conventional LOCO technique and close to the estimated betabeating limit (about $0.4 \%$ ) determined by systematic errors of the LOCO algorithm and power supply stability limit. In Fig. 9, the error bars represent the standard deviation of 10 repeated measurements. The residual beta-beat is significantly larger than the measurement errors.

The same crosscheck has been done to compare the dispersion correction by different methods. The results are presented in Fig. 10 showing the residual rms errors of horizontal (left) and vertical (right) dispersion $\Delta \eta=\eta_{\text {meas }}-$ $\eta_{\text {model }}$ plotted versus the number of iterations. Similarly,
AC LOCO reduces the dispersion errors significantly compared to DC LOCO and to the TbT-based techniques.

\section{CONCLUSION}

A fast and precise $\mathrm{AC}$ LOCO technique of magnet lattice correction has been developed. AC LOCO is based on the ORM measurement with sine-wave excitation of the beam using fast correctors. The conventional LOCO at NSLS-II takes $1 \mathrm{~h}$ for one iteration of the lattice correction, and achieves a measurement precision of $1 \mu \mathrm{m}$. In contrast, AC LOCO achieves a measurement precision of $15 \mathrm{~nm}$, and it takes only a few minutes. The significantly improved accuracy of the ORM measurement results in a reduction in the residual beta function errors of the corrected linear lattice by a factor of two. The correction accuracy is no longer limited by the measurement but rather by the LOCO algorithm itself and by the power supply stability. Further reduction of the measurement time is achieved by driving multiple correctors with different frequencies simultaneously. In the case of 30 correctors, one AC LOCO measurement takes about as much time as TbT-based techniques (about $1 \mathrm{~min}$ ), while offering 100 times better precision. At NSLS-II, we have carried out an experiment of the 
multifrequency excitation of 23 correctors. We were able to keep the measurement accuracy in the nanometer level for all the excitation frequencies in the $10-54 \mathrm{~Hz}$ range. Based on the LOCO simulation results, the achieved high precision could open the door for finding sextupole alignment errors.

\section{ACKNOWLEDGMENTS}

This work was supported by the U.S. Department of Energy under Contract No. DE-AC02-98CH10886.

[1] J. Safranek, Experimental determination of storage ring optics using closed orbit response measurements, Nucl. Instrum. Methods Phys. Res., Sect. A 388, 27 (1997).

[2] A. W. Chao and M. Tigner, Handbook of Accelerator Physics and Engineering (World Scientific, Singapore, 1999).

[3] V. Smaluk et al., in Proceedings of IPAC'16, Busan (2016), http://accelconf.web.cern.ch/accelconf/ipac2016/ papers/thpmr008.pdf.
[4] I. P. S. Martin et al., in Proceedings of IPAC'14, Dresden (2014), http://accelconf.web.cern.ch/accelconf/IPAC2014/ papers/tupri083.pdf.

[5] Y. Tian and L. H. Yu, in Proceedings of PAC'11, New York (2011), http://accelconf.web.cern.ch/accelconf/pac2011/ papers/weodn4.pdf.

[6] K. Vetter et al., in Proceedings of BIW'12, Newport News (2012), https://accelconf.web.cern.ch/accelconf/BIW2012/ papers/wecp02.pdf.

[7] W. Cheng et al., in Proceedings of IBIC'15, Melbourne (2015), https://accelconf.web.cern.ch/AccelConf/IBIC2015/ papers/thala02.pdf.

[8] G. M. Wang et al., BNL Tech. Note No. 168, 2015.

[9] X. Huang, S. Y. Lee, E. Prebys, and R. Tomlin, Application of independent component analysis to Fermilab booster, Phys. Rev. ST Accel. Beams 8, 064001 (2005).

[10] J. Irwin, C. X. Wang, Y. T. Yan, K. L. F. Bane, Y. Cai, F.-J. Decker, M. G. Minty, G. V. Stupakov, and F. Zimmermann, Model-Independent Beam Dynamics Analysis, Phys. Rev. Lett. 82, 1684 (1999).

[11] Y. Hidaka, B. Podobedov, and J. Bengtsson, in Proceedings of NA-PAC'16, Chicago (2016), http://vrws.de/ napac2016/papers/tupob52.pdf. 